\title{
Clinical utility of thoracic endosonography (EBUS/EUS-b) in mediastinal staging of patients with non-small cell lung cancer: comparison with integrated PET/CT - a real-life prospective study in Greece
}

\author{
Serafeim Chrysikos ${ }^{1}$, Ioannis Gkiozos ${ }^{2}$, Katerina Dimakou ${ }^{1}$, Eleftherios Zervas ${ }^{3}$, Theodoros Karampitsakos ${ }^{1}$, \\ Maria Anyfanti ${ }^{3}$, Argyrios Tzouvelekis ${ }^{4}$, Konstantinos Samitas ${ }^{3}$, Mina Gaga ${ }^{3}$, Nikolaos Koulouris ${ }^{4}$, \\ Ioannis Vasileiadis ${ }^{5}$, Konstantinos Syrigos ${ }^{2}$
}

${ }^{1} 5^{\text {th }}$ Respiratory Medicine Department, "Sotiria" Chest Diseases Hospital, Athens, Greece; ${ }^{2} 3^{\text {rd }}$ Internal Medicine Department, "Sotiria" Chest Diseases Hospital, Medical School, National and Kapodistrian University of Athens, Athens, Greece; ${ }^{3} 7^{\text {th }}$ Respiratory Medicine Department, Athens "Sotiria" Chest Diseases Hospital, Athens, Greece; ${ }^{4}{ }^{\text {st }}$ Academic Respiratory Medicine Department, "Sotiria" Chest diseases hospital, Medical School, National and Kapodistrian University of Athens, Athens, Greece; ${ }^{5}$ Intensive Care Unit, First Department of Respiratory Medicine, Medical School, National and Kapodistrian University of Athens, Athens, Greece

Contributions: (I) Conception and design: S Chrysikos, I Gkiozos, T Karampitsakos, E Zervas, K Samitas, I Vaseiliadis, K Syrigos; (II) Administrative support: S Chrysikos, I Gkiozos, K Dimakou, E Zervas, T Karampitsakos, A Tzouvelekis, M Gaga N Koulouris, I Vasileiadis, K Syrigos; (III) Provision of study materials or patients: S Chrysikos, E Zervas, K Samitas ; (IV) Collection and assembly of data: S Chrysikos, I Gkiozos, E Zervas, T Karampitsakos, M Anyfanti, I Vasileiadis, K Syrigos; (V) Data analysis and interpretation: All authors; (VI) Manuscript writing: All authors; (VII) Final approval of manuscript: All authors.

Correspondence to: Serafeim Chrysikos. 5th Respiratory Medicine Department, "Sotiria" Chest Diseases Hospital, Mesogeion Avenue 152, 11527 Athens, Greece. Email: makischr@hotmail.com.

Background: Accurate mediastinal staging in patients with non-small cell lung cancer (NSCLC) is crucial for the determination of optimal treatment management.

Methods: This was a real-life prospective study enrolling 140 patients between December 2016 and August 2018. We aimed to determine the clinical utility of EBUS/EUS-b in mediastinal staging of patients with NSCLC in comparison with integrated PET/CT. Furthermore, $\mathrm{SUV}_{\max }$ cut-off value with the highest specificity/accuracy was evaluated. Subgroup analysis according to histological type was performed.

Results: One hundred and thirty patients were eligible for analysis (mean age \pm SD: 67.6 \pm 7.6, males 97). Three hundred different lymph node stations were sampled (272 through EBUS-TBNA and 28 through EUS-b FNA). Mean SUV max $_{\text {max }}$ of all malignant lymph nodes was 7.46 (SD =5.54). Sensitivity, specificity, PPV and NPV of EBUS/EUS-b for the identification of mediastinal malignant lymph nodes was $93.8 \%, 100 \%$, $100 \%$, and $93.4 \%$, respectively. Accordingly, PET/CT yielded $92.2 \%$ sensitivity, $43.9 \%$ specificity, $64.8 \%$ PPV and $83.3 \%$ NPV. For adenocarcinoma (n=76) NPV were $86.2 \%$ with EBUS/EUS-b and $75 \%$ with PET/CT. NPV for squamous cell $(\mathrm{n}=46)$ was $100 \%$ with EBUS/EUS-b and $90.9 \%$ with PET/CT. EBUS/ EUS-b staging yielded excellent agreement with final staging $(97.5 \%$, Tau $0.94, \mathrm{P}<0.001)$. ROC curve analysis identified the value 4.95 as the optimal $\mathrm{SUV}_{\max }$ cut-off value with the best specificity (87.4\%) and accuracy (79\%) (AUC 0.69; 95\% CI: 0.73-0.84, P<0.001).

Conclusions: Thoracic endosonography is an excellent, minimally invasive tool yielding high sensitivity and diagnostic accuracy in mediastinal staging of patients with NSCLC. Implementation of both EBUS/ EUS-b and PET/CT is necessary before any surgical intervention.

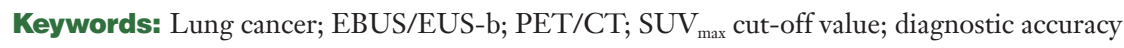


Submitted Apr 23, 2020. Accepted for publication Aug 26, 2020.

doi: $10.21037 /$ jtd-20-1735

View this article at: http://dx.doi.org/10.21037/jtd-20-1735

\section{Introduction}

Lung cancer represents the main cause of cancer-related deaths worldwide leading to approximately 1.4 million deaths per year and a 5-year relative survival rates for non-small cell lung cancer (NSCLC) based on stage (localized, regional or distant) to be $61 \%, 35 \%$, and $6 \%$ respectively (1). Only $16 \%$ of patients with NSCLC are diagnosed in early stages (N0-N1 disease) and gain benefit from surgical lung resections. Most patients are diagnosed with a systematic spread of the disease via lymphatics to the mediastinal lymph nodes (N2-N3 disease) or via the bloodstream to the contralateral lung or distant organs, rendering them inoperable (M1 disease). Accurate clinical staging of the mediastinum is of paramount importance to determine proper treatment and limit the number of futile thoracotomies (2-7). There are two main methods of mediastinal staging: (I) non-invasive methods [chest computed tomography (CT), positron-emission tomography (PET) and integrated PET/CT $(3,8)]$ and (II) invasive methods [transthoracic needle aspiration (TTNA), endobronchial ultrasound-guided transbronchial needle aspiration (EBUS-TBNA) alone or combined with transesophageal bronchoscopic ultrasound-guided fineneedle aspiration (EUS-b FNA), and mediastinoscopy]. In the context of CT, every lymph node with a short-axis diameter $>1 \mathrm{~cm}$ is considered malign. Previous studies have shown that this cut-off yielded sensitivity, specificity, positive predictive value (PPV), and negative predictive value (NPV) of $55 \%, 81 \%, 58 \%$ and $87 \%$, respectively $(3,9)$. PET is a functional imaging modality using a radioanalog F-18 fluorodeoxyglucose (FDG), which is taken up by hypermetabolic malignant cells. We must highlight that the role of PET in the staging of NSCLC (per guidelines) is not mainly related to mediastinum but to exclude distant metastasis. Studies have shown that cut-off levels of maximum standard uptake value $\left(\mathrm{SUV}_{\text {max }}\right) \geq 2.5$ can predict malignancy with a sensitivity, specificity, PPV, NPV of $80 \%$, $88 \%, 75 \%$, and $91 \%$ respectively $(3,10,11)$. Low specificity of the method is mainly attributed to false-positive FDG uptake of lymph nodes due to infectious and inflammatory processes $(3,10,12)$. The role of integrated PET/CT is steadily increasing, with a significant impact on the overall survival of patients with NSCLC $(3,13)$. Prediction accuracy of TNM staging has been optimized with imaging modalities; yet discrepancies with pathology features often encounter. Therefore, according to guidelines, histologic verification of enlarged or hypermetabolic lymph nodes is recommended $(3,4,12)$. Currently, minimally invasive techniques such as EBUS-TBNA alone, or combined with EUS-b FNA have widely used in biopsy sampling most mediastinal lymph node stations except the paraaortic and pre-vascular ones. On the other hand, surgical interventions, including mediastinoscopy, require general anesthesia and harbor high operative risk (14-21). EBUSTBNA has demonstrated superiority compared to CT and PET/CT in several studies with sensitivity, specificity, PPV and NPV of $89 \%, 100 \%, 100 \%$, and $91 \%$, respectively (8,22-24). Furthermore, combined EBUS/EUS-b augment diagnostic values of sensitivity and NPV to $91 \%$ and $96 \%$ respectively establishing this technique according to recent guidelines as one reliable option for evaluation of the mediastinum in potentially operable NSCLC patients (25-30). The primary aim of this real-life prospective study was to determine the clinical utility of EBUS/EUS-b in mediastinal staging of patients with NSCLC compared to integrated PET/CT. Furthermore, to evaluate the $\mathrm{SUV}_{\text {max }}$ cut-off value with the highest specificity/accuracy.

\section{Methods}

\section{Study design/patient selection}

In this prospective study, we enrolled 140 patients with histologically proven $(\mathrm{n}=12)$ or radiologically suspected $(\mathrm{n}=128)$ potentially operable NSCLC (clinical stage I-III) who presented in 2 different respiratory departments in "Sotiria" General Hospital for Thoracic Diseases, Athens, Greece, from December 2016 to August 2018. All of them underwent EBUS-TBNA alone or combined with EUS-b FNA with diagnosis and staging of NSCLC to be the main indication for the procedure. Histologically proven patients with NSCLC through simple bronchoscopy or TTNA were referred to our hospital from other centers where EBUS/ EUS-b was not available. Before EBUS/EUS-b procedure, a chest CT scan with contrast to assess resectability of 
the primary tumor and evaluation of the mediastinal/hilar lymph node size was available. Furthermore, integrated PET/CT in the same PET center and brain CT or brain imaging with contrast were performed to evaluate the hypermetabolic activity of mediastinal/hilar lymph nodes and exclude distal metastasis. Lymph nodes with shortaxis $>1 \mathrm{~cm}$ on $\mathrm{CT}$ and $\mathrm{SUV}_{\max } \geq 2.5$ on FDG-PET/CT were interpreted as positive. The results from chest CT and PET/CT (lymph nodes size and $\mathrm{SUV}_{\max }$ value) were compared with histology results obtained from EBUS/ EUS-b. In patients with EBUS/EUS-b negative N2/N3 disease was recommended further surgery exploration (thoracotomy). Subgroup analysis, according to histological type, was performed. The clinical stage (c TNM) obtained from PET/CT and EBUS/EUS- $b$, and final stage ( $p$ TNM) obtained from EBUS/EBUS-b and postoperative results were done according to the latest $8^{\text {th }}$ TNM classification (7). Patients medically inoperable or patients with Pancoast (apical) tumor, involvement of supraclavicular nodes (N3 disease), T3 or T4 tumor with mediastinal invasion or presence of metastatic M1 disease, as well as patients with small cell lung cancer, lymphoproliferative malignancies or other causes of mediastinal lymphadenopathy (i.e., sarcoidosis/tuberculosis) were excluded from the analysis. This prospective study was approved by the institutional review board (IRB protocol approval: 23960/02.12.16), and written informed consent was obtained from all patients. The study was conducted in accordance with the Declaration of Helsinki (as revised in 2013).

\section{EBUS-TBNA and EUS-b FNA procedures}

EBUS-TBNA was performed using a flexible convex probe ultrasound bronchoscope (BF-UC180F, OLYMPUS, Tokyo, Japan). Two trained interventional pulmonologists (SC, EZ) performed all EBUS/EUS-b procedures. Systematic evaluation of mediastinal and hilar lymph nodes visible by EBUS was performed. The short-axis diameter of every detected node was recorded. Nodes with short-axis diameter $\geq 5 \mathrm{~mm}$ were sampled using a dedicated 21-G EBUS-TBNA needle. We attempted to obtain at least three samples from each node. $\mathrm{N} 3$ nodes were sampled first and then N2 and N1 nodes to avoid cross-contamination. EUS-b FNA was performed for those nodes that were inaccessible or technically difficult to access by EBUS-TBNA, such as left lower paratracheal (4L), paraesophageal (8) and pulmonary ligament lymph node station (9). Following sampling, aspirated material was dispersed onto glass slides, smeared, fixed in $95 \%$ alcohol, and stained with hematoxylin-eosin (HE) and Papanicolau. Tissue cores obtained by EBUS/ EUS-b were fixed with $10 \%$ formalin and stained using HE. Rapid on-site evaluation (ROSE) was not available in our center.

\section{Treatment}

Patients with histologic confirmation of N2/N3 disease through EBUS/EUS-b were referred to the oncology department of our institution for initiation of induction or radical chemotherapy +/- radiation. With regards to potentially operable patients with nonhistologic confirmation of $\mathrm{N} 2 / \mathrm{N} 3$ disease after thoracic endosonography, we recommended open thoracotomy or Video Assistant Thoracic Surgery (VATS) lobectomy with complete lymph node dissection.

\section{Statistical analysis}

ROC curve analysis was used to find the optimal $\mathrm{SUV}_{\max }$ cut-off for the prediction of lymph node spread. The overall performance of the ROC analysis was quantified by computing the area under the curve (AUC). Using ROC analysis, optimal sensitivity and specificity were also determined. The accuracy of PET/CT and EBUS/ EUS-b were evaluated with the calculation of sensitivity, specificity, PPV, and NPV. In the context of the evaluation of concordance between PET/CT, EBUS/EUS-b, and surgical pathology regarding stage assessment, we used the Kendall's Tau coefficient. For the assessment of agreement between PET/CT, and EBUS/EUS-b for nodal status, Kappa coefficient $(\mathrm{K})$ was used. The significance value was set at $\mathrm{P}<0.05$. Statistical analyses were performed using SPSS 22.0 program.

\section{Results}

\section{Baseline characteristics and study design}

One hundred forty patients with proven $(n=12)$ or radiologically suspected $(n=128)$ NSCLC were initially registered in the study from December 2016 to August 2018. Ten out of 140 patients were excluded from the study following the EBUS/EUS-b procedure due to the diagnosis of small cell lung cancer $(n=7)$ or other benign conditions such as sarcoidosis or tuberculosis $(n=3)$. Demographic and 
Table 1 Demographics and clinical characteristics

\begin{tabular}{|c|c|}
\hline Characteristics & $\mathrm{N}(\%)$ \\
\hline Age, mean (SD) & $67.6(7.6)$ \\
\hline \multicolumn{2}{|l|}{ Gender } \\
\hline Men & $97(74.6)$ \\
\hline Women & $33(25.4)$ \\
\hline Smoking & 119 (91.5) \\
\hline \multicolumn{2}{|l|}{ Clinical stage } \\
\hline \multicolumn{2}{|l|}{ IA } \\
\hline PET-CT & $8(6.2)$ \\
\hline EBUS-EUS & 21 (16.2) \\
\hline \multicolumn{2}{|l|}{ IB } \\
\hline PET-CT & $4(3.1)$ \\
\hline EBUS-EUS & $10(7.7)$ \\
\hline \multicolumn{2}{|l|}{$\| A$} \\
\hline PET-CT & $3(2.3)$ \\
\hline EBUS-EUS & $11(8.5)$ \\
\hline \multicolumn{2}{|l|}{ IIB } \\
\hline PET-CT & $11(8.5)$ \\
\hline EBUS-EUS & $21(16.2)$ \\
\hline \multicolumn{2}{|l|}{ IIIA } \\
\hline PET-CT & $63(48.5)$ \\
\hline EBUS-EUS & 51 (39.2) \\
\hline \multicolumn{2}{|l|}{ IIIB } \\
\hline PET-CT & $37(28.5)$ \\
\hline EBUS-EUS & $14(10.8)$ \\
\hline \multicolumn{2}{|l|}{ IIIC } \\
\hline PET-CT & $4(3.1)$ \\
\hline EBUS-EUS & $2(1.5)$ \\
\hline \multicolumn{2}{|l|}{ Nodal stage } \\
\hline \multicolumn{2}{|l|}{ NO } \\
\hline PET-CT & 19 (14.6) \\
\hline EBUS/EUS & $59(45.4)$ \\
\hline \multicolumn{2}{|l|}{$\mathrm{N} 1$} \\
\hline PET-CT & $14(10.8)$ \\
\hline EBUS/EUS & $11(8.5)$ \\
\hline
\end{tabular}

Table 1 (Continued)
Table 1 (Continued)

\begin{tabular}{|c|c|}
\hline Characteristics & $\mathrm{N}(\%)$ \\
\hline \multicolumn{2}{|l|}{ N2 } \\
\hline PET-CT & $62(47.7)$ \\
\hline EBUS/EUS & $46(35.4)$ \\
\hline \multicolumn{2}{|l|}{ N3 } \\
\hline PET-CT & $35(26.9)$ \\
\hline EBUS/EUS & $14(10.8)$ \\
\hline \multicolumn{2}{|l|}{ Final diagnosis } \\
\hline Adenocarcinoma & $76(58.5)$ \\
\hline Squamous cell carcinoma & $46(35.4)$ \\
\hline NSCLC-NOS & $8(6.2)$ \\
\hline \multicolumn{2}{|l|}{ Final stage } \\
\hline I & $26(21.5)$ \\
\hline II & $25(20.7)$ \\
\hline III & $70(57.9)$ \\
\hline Mass SUV max (PET-CT), median (IQR) & $13.5(7.7-18)$ \\
\hline $\begin{array}{l}\text { Size (short-axis) of lymph nodes in } \mathrm{cm} \\
\text { (PET-CT), median (IQR) }\end{array}$ & $1(0.8-1.3)$ \\
\hline $\begin{array}{l}\text { Size (short-axis) of lymph nodes in cm } \\
\text { (EBUS/EUS-b), median (IQR) }\end{array}$ & $1(0.7-1.4)$ \\
\hline \multicolumn{2}{|l|}{ Location of LN's sampled } \\
\hline LN10-11R & $62(47.7)$ \\
\hline LN4R & $65(50.0)$ \\
\hline LN7 & $93(71.5)$ \\
\hline LN2R & $2(1.5)$ \\
\hline LN2L & $2(1.5)$ \\
\hline LN4L & $38(29.2)$ \\
\hline LN10-11L & $36(27.7)$ \\
\hline LN8-9 & $2(1.5)$ \\
\hline
\end{tabular}

clinical characteristics of the study population are shown in Table 1. Study design is shown in Figure 1. Application of PET/CT before EBUS/EUS-b procedure characterized $9.3 \%$ of patients as clinical stage I, $10.8 \%$ as stage II, $80 \%$ as stage III, and was indicative of N2/N3 disease in 97 patients (74.6\%). Mean $\mathrm{SUV}_{\max }$ uptake value of recorded hypermetabolic lymph nodes $(\mathrm{n}=266)$ was $6.03(\mathrm{SD}=5.40)$. 


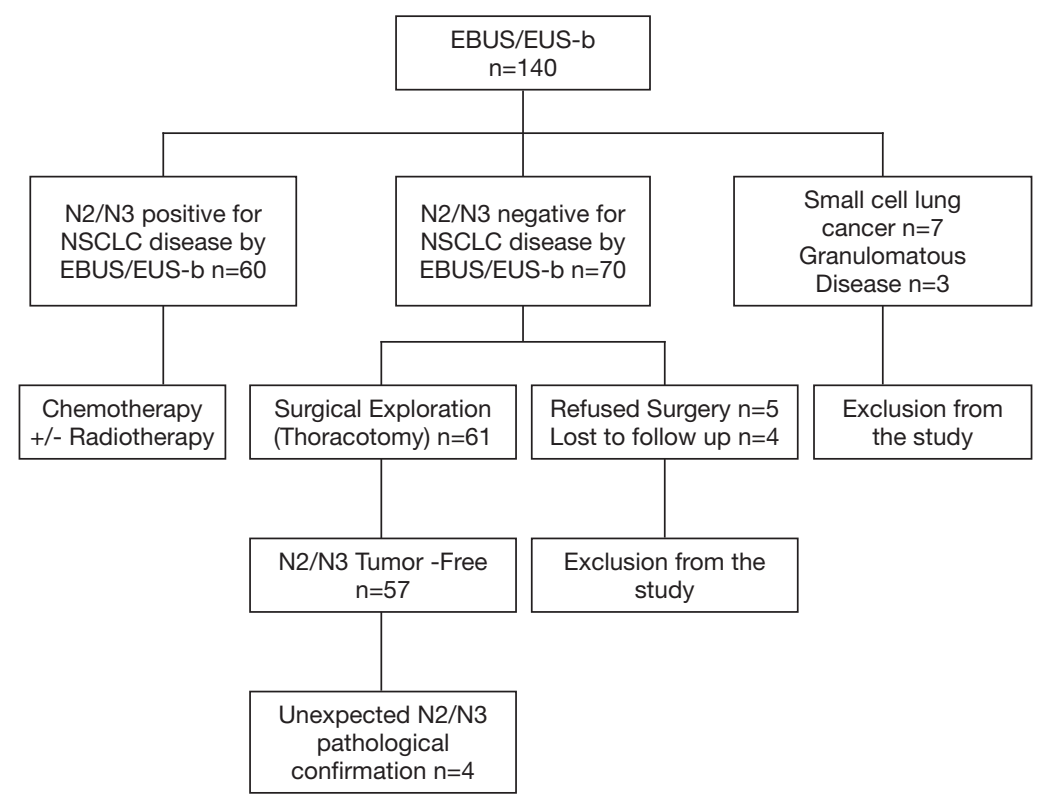

Figure 1 Flowchart of patients enrolling in the study.

Median size (short-axis) of lymph nodes recorded both from chest CT and EBUS/EUS-b was $1 \mathrm{~cm}$ (range, 0.7-1.40). EBUS/EUS-b led to a diagnosis of NSCLC in $81 / 130$ patients $(62.3 \%)$. Adenocarcinoma was the final diagnosis in $58.5 \%$ of the study group, squamous cell carcinoma in $35.4 \%$, and NSCLC-Not Otherwise Specified (NOS) in $6.2 \%$. Most patients were finally classified as stage III $(43 \%$ IIIA, $13.2 \%$ IIIB, 1.7\% IIIC). Pathologic confirmation of N2/N3 disease through EBUS/EUS-b and postoperative histology was established in $52.9 \%$ of patients.

\section{EBUS/EUS- $b$}

A total of 300 lymph nodes were sampled (272 through EBUS-TBNA and 28 through EUS-b FNA). Table 1 shows that most frequently sampled lymph nodes were subcarinal (7), right paratracheal (4R), right hilar (10$11 \mathrm{R})$ and left paratracheal (4L) (71.5\%, 50\%, 47.7\% and $29.2 \%$ respectively). EBUS/EUS-b was indicative of N2/ $\mathrm{N} 3$ disease in $60 / 130$ patients $(46.2 \%)$. In the remaining 70 patients, further surgical exploration was recommended. In 9 patients, no surgical verification was performed because of the patient's refusal $(n=5)$ or loss of follow-up $(n=4)$ and were excluded from the study. EBUS/EUS-b, in comparison with postoperative results for nodal spread, demonstrated four false-negative cases. All of them involved adenocarcinoma in the histologic subtype. Furthermore,
EBUS/EUS-b confirmed occult mediastinal involvement in 3/33 PET/CT scan negative mediastinal cases (2 with adenocarcinoma and 1 with squamous cell carcinoma). No severe complications in terms of pneumothorax or clinically significant bleeding were observed during the study period.

\section{Diagnostic value of FDG-PET/CT SUV $V_{\max }$}

Mean $\mathrm{SUV}_{\max }$ of all malignant lymph nodes was 7.46 (SD $=5.54$ ), and the mean $\mathrm{SUV}_{\text {max }}$ of all benign lymph nodes was $2.99(\mathrm{SD}=5.15)(\mathrm{P}<0.001)$. When a $\mathrm{SUV}_{\max }$ value of 2.5 was used, the sensitivity, specificity, PPV, NPV, and accuracy were $82 \%, 43.7 \%, 53.7 \%, 75.3 \%$, and $60.7 \%$, respectively. False-negative and false-positive rates based on FDG-PET/ $\mathrm{CT} \mathrm{SUV}_{\max }$ and EBUS/EUS-b pathology results were $8 \%$ and $31 \%$, respectively.

ROC analysis (Figure 2) showed that the optimal $S_{U} V_{\max }$ cut-off value with the highest accuracy for predicting malignant nodes through EBUS/EUS-b was 4.95 with $68.4 \%$ sensitivity, $87.4 \%$ specificity, $81.3 \%$ PPV, $77.7 \%$ NPV and $79 \%$ accuracy. The AUC was 0.69 .

\section{Diagnostic value of lymph node size}

Median size (short-axis) of all malignant lymph nodes both from chest CT and EBUS/EUS-b was $1.2 \mathrm{~cm}$ (range, 1-1.6), while the median size of all benign lymph nodes was 
$0.9 \mathrm{~cm}$ (range, $0.7-1.2)(\mathrm{P}<0.001)$. Sensitivity, specificity, PPV, NPV, and accuracy of chest CT for the detection of malignant lymph nodes when the short diameter is defined as $1 \mathrm{~cm}$ were $51.87 \%, 83.23 \%, 71.13 \%, 68.47 \%$, and $69.33 \%$, respectively. The false negative rate based on lymph node size on CT and EBUS/EUS-b pathology results was $21 \%$.

\section{Clinical versus final pathologic stage}

PET/CT was associated with $71.9 \%$ agreement of clinical and pathological stage (Tau coefficient $0.52, \mathrm{P}<0.001$ ). EBUS/EUS-b was associated with $97.5 \%$ agreement (Tau coefficient $0.94, \mathrm{P}<0.001$ ) (Table 2). Furthermore, there

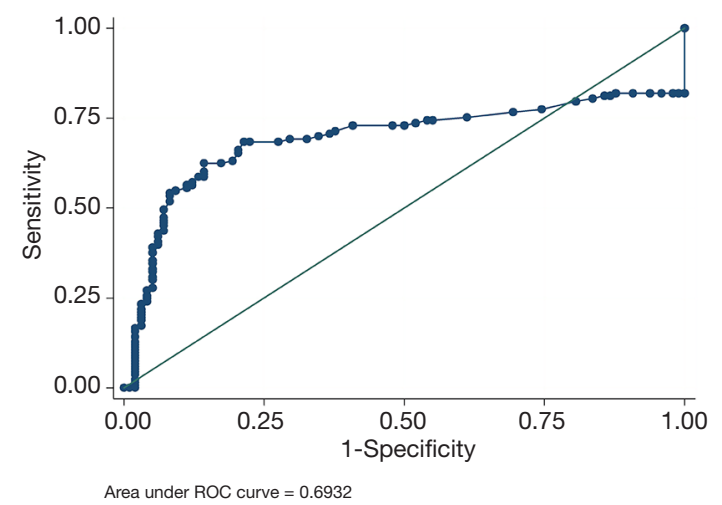

Figure 2 ROC analysis showed that optimal SUV $\mathrm{max}_{\text {max }}$ cut-off value with the highest accuracy for predicting malignant nodes through EBUS/EUS-b was 4.95 with $68.4 \%$ sensitivity, $87.4 \%$ specificity, $81.3 \%$ PPV, $77.7 \%$ NPV and $79 \%$ accuracy. The AUC was 0.69. was excellent agreement between EBUS/EUS-b and pathological confirmation of nodal status (96.7\%; kappa 0.93, $\mathrm{P}<0.001)$ and poor agreement between PET/CT and pathological confirmation of nodal status (71.9\%, kappa 0.37, $\mathrm{P}<0.001)$. EBUS/EUS-b managed to down-stage disease in 40 patients $(30.8 \%)$ and up staged disease in 4 cases $(3.1 \%)$.

\section{Comparative diagnostic accuracy of combined EBUS/ EUS- $b$ and integrated PET-CT in mediastinal staging of NSCLC}

Table 3 shows the diagnostic value of integrated PET/ CT and EBUS/EUS-b in the evaluation of mediastinal lymph node staging both in total sample and based on different types of carcinoma. EBUS/EUS-b demonstrated a sensitivity, specificity, PPV, NPV and accuracy $93.8 \%$, $100 \%, 100 \%, 93.4 \%$, and $96.69 \%$ respectively. The respective values for $\mathrm{PET} / \mathrm{CT}$ were $92.2 \%, 43.9 \%$, $64.8 \%, 83.3 \%$ and $72.72 \%$. Based on histologic subgroup analysis, EBUS/EUS-b yielded a $91.1 \%$ sensitivity and $86.2 \%$ NPV for adenocarcinoma and both $100 \%$ for squamous cell carcinoma. Accordingly, PET/CT yielded a 91.1\% sensitivity and 75\% NPV for adenocarcinoma and $93.3 \%$ sensitivity and $90.9 \%$ specificity for squamous cell carcinoma.

\section{Discussion}

To the best of our knowledge, this is the first real-life prospective study in Greece, showing that EBUS/EUS-b

Table 2 Comparison of final stage obtained from postoperative and EBUS/EUS-b histologic results with clinical stage obtained from PET-CT and EBUS/EUS-b

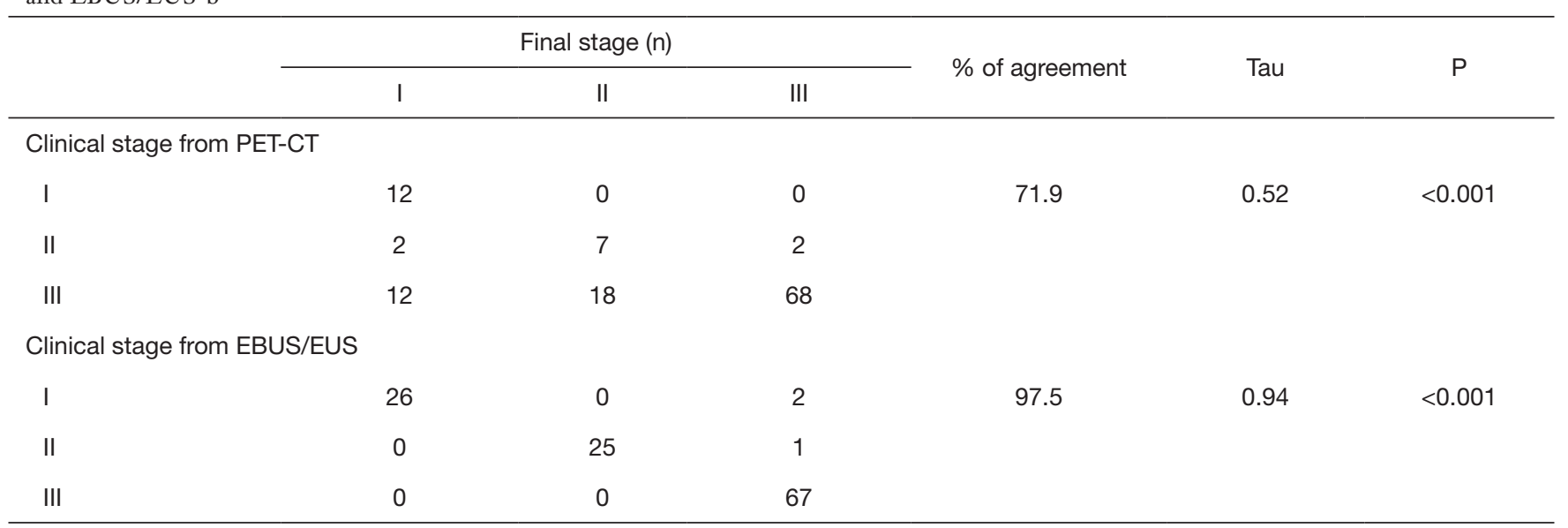


Table 3 Sensitivity, specificity, positive and negative predictive value for the prediction of malignant mediastinal (N2/N3) lymph nodes from PET/CT and EBUS/EUS-b

\begin{tabular}{|c|c|c|}
\hline Patient groups & PET-CT & EBUS/EUS-b \\
\hline Sensitivity (95\% Cl) & $92.2(82.7-97.4)$ & $93.8(84.8-98.3)$ \\
\hline Specificity $(95 \%$ Cl) & $43.9(30.7-57.6)$ & $100(93.7-100)$ \\
\hline PPV (95\% Cl) & 64.8 (54.1-74.6) & $100(94.0-100)$ \\
\hline \multicolumn{3}{|l|}{ Adenocarcinoma } \\
\hline Sensitivity $(95 \% \mathrm{Cl})$ & $91.1(78.8-97.5)$ & $91.1(78.8-97.5)$ \\
\hline Specificity (95\% Cl) & $48.0(27.8-68.7)$ & $100(86.3-100)$ \\
\hline PPV (95\% Cl) & 75.9 (62.4-86.5) & $100(91.4-100)$ \\
\hline Sensitivity $(95 \% \mathrm{Cl})$ & $93.3(68.1-99.8)$ & $100(78.2-100)$ \\
\hline Specificity $(95 \% \mathrm{Cl})$ & 35.7 (18.6-55.9) & $100(87.7-100)$ \\
\hline PPV (95\% Cl) & $43.8(26.4-62.3)$ & $100(78.2-100)$ \\
\hline NPV (95\% Cl) & $90.9(58.7-99.8)$ & $100(87.7-100)$ \\
\hline \multicolumn{3}{|l|}{ NSCLC-NOS } \\
\hline Sensitivity (95\% Cl) & $100(39.8-100)$ & $100(39.8-100)$ \\
\hline Specificity (95\% Cl) & 75.0 (19.4-99.4) & $100(39.8-100)$ \\
\hline PPV (95\% Cl) & $80.0(28.4-99.5)$ & $100(39.8-100)$ \\
\hline
\end{tabular}

had better diagnostic accuracy in mediastinal staging of patients with NSCLC compared to integrated PET/CT. Sensitivity rates were similar; yet, the specificity of EBUS/ EUS-b for malignancy was significantly higher than that of PET/CT. PPV of EBUS for malignancy was higher than that of PET/CT, while the NPV of both methods was similar.

In the present study, EBUS/EUS-b confirmed occult mediastinal involvement in 3/33 PET/CT scan negative mediastinal cases ( 2 with adenocarcinoma and 1 with squamous cell carcinoma). These results are in line with existing published studies $(3,31)$ supporting the theory that EBUS/EUS-b has great potential even in patients with no abnormal mediastinum uptake on PET/CT.

Our study yielded similar results with previously published literature $(22,23,26,28,32)$. In particular, the reported sensitivity and specificity of integrated PET/CT for the prediction of malignant $\mathrm{N} 2 / \mathrm{N} 3$ lymph nodes ranged from $61 \%$ to $89 \%$ and from $59 \%$ to $96 \%$, respectively $(3,9,10,13,26)$. In our study, sensitivity was $92.2 \%$ and specificity $43.9 \%$. High sensitivity of integrated PET/ CT in the present study could be attributed to the study design (33/130 of PET/CT scan cases with no abnormal mediastinum uptake). Low specificity of PET/CT in our study as well as in other studies maybe due to high falsepositive rates. In our study, false-negative ( $8 \%$ ) and falsepositive rates $(31 \%)$ based on FDG-PET/CT SUV max $_{\text {ax }}$ and EBUS/EUS-b pathology results were similar to previous studies ranging from $5-7 \%$ and $16-22 \%$, respectively.

With regards to the diagnostic value of lymph node size in chest $\mathrm{CT}$, our results are comparable with previous data showing that lymph nodes with short-axis $>1 \mathrm{~cm}$ had $55 \%$ sensitivity, $81 \%$ specificity, $58 \% \mathrm{PPV}$, and $87 \% \mathrm{NPV}$ for malignancy $(3,9)$. Thus, our findings further corroborate 
the evidence that chest CT is inferior to integrated PET/ CT for malignancy detection (3). In the present study, our lymph node size false-negative rate $(21 \%)$ was similar to previously published studies $(3,33,34)$. This proves that all nodes $\geq 5 \mathrm{~mm}$ should be sampled, especially in $4 \mathrm{~L}, 7$, and $4 \mathrm{R}$ locations as well if it is known to be adenocarcinoma $(31,35)$.

Data derived from the literature showed that $\mathrm{SUV}_{\text {max }}$ value $\geq 2.5$ had sensitivity, specificity, PPV, NPV of $80 \%$, $88 \%, 75 \%$, and $91 \%$ for the detection of lymph node malignancy, respectively (3). In our study, $\mathrm{SUV}_{\max }$ value $\geq 2.5$ had $82 \%$ sensitivity, $43.7 \%$ specificity, $53.7 \%$ PPV, $75.3 \%$ NPV and $60.7 \%$ accuracy for malignancy. Furthermore, we found through ROC analysis 4.95 as the $\mathrm{SUV}_{\text {max }}$ cutoff value with the higher diagnostic accuracy for the discrimination between benign and malignant nodes. This finding is in line with previous studies reporting as optimal cut-off values ranging from 4 to $5.3(36,37)$.

Finally, thoracic endosonography staged NSCLC with higher precision compared to integrated PET/CT. Percent agreement between the clinical stage by EBUS/EUS-b and final stage was excellent, while the respective percent agreement with PET/CT was moderate. Importantly, EBUS/EUS-b managed to down-stage the disease in 40 patients (30.8\%).

Our study should be interpreted in the context of some limitations. At first, heterogeneity of our study group, involving patients from clinical stage I to stage III, might have influenced diagnostic rates. Secondly, the lack of ROSE in our study may influence underestimating true positive rates by EBUS/EUS-b. Finally, the utility of EUS-b was restricted only in lymph nodes that were inaccessible or technically difficult to access by EBUSTBNA or to enlarged and FDG-PET/CT-avid lower mediastinal lymph nodes. On the other hand, these are issues characterizing a real-life prospective study. Despite the real-life setting, we managed to provide results from a large cohort with minimal missing data, and this confers significant consistency to the findings presented.

\section{Conclusions}

Our prospective study is in line with previous studies which confirm that thoracic endosonography is an excellent, minimally invasive tool yielding high sensitivity and diagnostic accuracy in mediastinal staging of patients with potentially operable NSCLC. Therefore, according to recent guidelines, the combination of EBUS/EUS-b following integrated PET/CT is strongly recommended before any surgical intervention. Although 4.95 is a cut-off value with the highest specificity and diagnostic accuracy, $\mathrm{SUV}_{\text {max }}$ cut-off value $\geq 2.5$ remains a safer and more practical cut-off value in everyday clinical practice in order not to exclude any patient for proper staging.

\section{Acknowledgments}

Funding: None.

\section{Footnote}

Data Sharing Statement: Available at http://dx.doi. org/10.21037/jtd-20-1735

Conflicts of Interest: All authors have completed the ICMJE uniform disclosure form (available at http://dx. doi. org/10.21037/jtd-20-1735). The authors have no conflicts of interest to declare.

Ethical Statement: The authors are accountable for all aspects of the work in ensuring that questions related to the accuracy or integrity of any part of the work are appropriately investigated and resolved. This prospective study was approved by the institutional review board of "Sotiria" Chest Disease hospital, Athens, Greece (IRB protocol approval: 23960/02.12.16) and written informed consent was obtained from all patients. The study was conducted in accordance with the Declaration of Helsinki (as revised in 2013).

Open Access Statement: This is an Open Access article distributed in accordance with the Creative Commons Attribution-NonCommercial-NoDerivs 4.0 International License (CC BY-NC-ND 4.0), which permits the noncommercial replication and distribution of the article with the strict proviso that no changes or edits are made and the original work is properly cited (including links to both the formal publication through the relevant DOI and the license). See: https://creativecommons.org/licenses/by-nc-nd/4.0/.

\section{References}

1. Jemal A, Bray F, Center MM, et al. Global cancer statistics. CA Cancer J Clin 2011;61:69-90.

2. Detterbeck FC, Postmus PE, Tanoue LT. The Stage Classification of Lung Cancer: Diagnosis and Management of Lung Cancer, 3rd ed: American College of Chest 
Physicians Evidence-Based Clinical Practice Guidelines. Chest 2013;143:e191S-e210S.

3. Silvestri GA, Gonzalez AV, Jantz MA, et al. Methods for Staging Non-small Cell Lung Cancer: Diagnosis and Management of Lung Cancer, 3rd ed: American College of Chest Physicians Evidence-Based Clinical Practice Guidelines. Chest 2013;143:e211S-50S.

4. Vansteenkiste J, De Ruysscher D, Eberhardt WE, et al. Early and locally advanced non-small-cell lung cancer (NSCLC): ESMO Clinical Practice Guidelines for diagnosis, treatment and follow-up. Ann Oncol 2013;24 Suppl 6:vi89-vi98.

5. Spira A, Ettinger DS. Multidisciplinary Management of Lung Cancer. N Engl J Med 2004;350:379-92.

6. Mountain CF, Dresler CM. Regional Lymph Node Classification for Lung Cancer Staging. Chest 1997;111:1718-23.

7. Detterbeck FC, Boffa DJ, Kim AW, et al. The Eighth Edition Lung Cancer Stage Classification. Chest 2017;151:193-203.

8. Rintoul RC, Tournoy KG, El Daly H, et al. EBUSTBNA for the Clarification of PET Positive IntraThoracic Lymph Nodes-an International Multi-Centre Experience. J Thorac Oncol 2009;4:44-8.

9. Toloza EM, Harpole L, McCrory DC. Noninvasive Staging of Non-small Cell Lung Cancer*: A Review of the Current Evidence. Chest 2003;123:137S-46S.

10. Pieterman RM, van Putten JWG, Meuzelaar JJ, et al. Preoperative Staging of Non-Small-Cell Lung Cancer with Positron-Emission Tomography. N Engl J Med 2000;343:254-61.

11. Cerfolio RJ, Ojha B, Bryant AS, et al. The role of FDGPET scan in staging patients with nonsmall cell carcinoma. Ann Thorac Surg 2003;76:861-6.

12. De Leyn P, Dooms C, Kuzdzal J, et al. Revised ESTS guidelines for preoperative mediastinal lymph node staging for non-small-cell lung cancer. Eur J Cardiothorac Surg 2014;45:787-98.

13. Lardinois D, Weder W, Hany TF, et al. Staging of NonSmall-Cell Lung Cancer with Integrated PositronEmission Tomography and Computed Tomography. N Engl J Med 2003;348:2500-7.

14. Toloza EM, Harpole L, Detterbeck F, et al. Invasive Staging of Non-small Cell Lung Cancer*: A Review of the Current Evidence. Chest 2003;123:157S-66S.

15. Gaga M, Chrysikos S, Bostantzoglou C, et al. Endobronchial Ultrasound-Guided Transbronchial Needle Aspiration/Biopsy (EBUS-TBNA/B): 5-Year
Experience of a Referral Center in Greece. In: A72 ADVANCES IN INTERVENTIONAL PULMONARY

[Internet]. American Thoracic Society; 2019 [cited 2019

Oct 27]. p. A2359-A2359. (American Thoracic Society International Conference Abstracts). Available online: https://www.atsjournals.org/doi/abs/10.1164/ajrccmconference.2019.199.1_MeetingAbstracts.A2359

16. Herth F, Becker HD, Ernst A. Conventional vs Endobronchial Ultrasound-Guided Transbronchial Needle Aspiration: A Randomized Trial. Chest 2004;125:322-5.

17. Wallace MB, Pascual JMS, Raimondo M, et al. Minimally Invasive Endoscopic Staging of Suspected Lung Cancer. JAMA 2008;299:540-6.

18. Yasufuku K, Chiyo M, Sekine Y, et al. Real-time Endobronchial Ultrasound-Guided Transbronchial Needle Aspiration of Mediastinal and Hilar Lymph Nodes. Chest 2004;126:122-8.

19. Yasufuku K, Chiyo M, Koh E, et al. Endobronchial ultrasound guided transbronchial needle aspiration for staging of lung cancer. Lung Cancer 2005;50:347-54.

20. Colella S, Vilmann P, Konge L, et al. Endoscopic ultrasound in the diagnosis and staging of lung cancer. Endosc Ultrasound 2014;3:205-12.

21. Czarnecka-Kujawa K, Yasufuku K. The role of endobronchial ultrasound versus mediastinoscopy for nonsmall cell lung cancer. J Thorac Dis 2017;9:S83-97.

22. Hwangbo B, Kim SK, Lee HS, et al. Application of Endobronchial Ultrasound-Guided Transbronchial Needle Aspiration Following Integrated PET/CT in Mediastinal Staging of Potentially Operable Non-small Cell Lung Cancer. Chest 2009;135:1280-7.

23. Yasufuku K, Nakajima T, Motoori K, et al. Comparison of Endobronchial Ultrasound, Positron Emission Tomography, and CT for Lymph Node Staging of Lung Cancer. Chest 2006;130:710-8.

24. Bauwens O, Dusart M, Pierard P, et al. Endobronchial ultrasound and value of PET for prediction of pathological results of mediastinal hot spots in lung cancer patients. Lung Cancer 2008;61:356-61.

25. Szlubowski A, Zieliński M, Soja J, et al. A combined approach of endobronchial and endoscopic ultrasoundguided needle aspiration in the radiologically normal mediastinum in non-small-cell lung cancer staging - a prospective trial. Eur J Cardiothorac Surg 2010;37:1175-9.

26. Hwangbo B, Lee GK, Lee HS, et al. Transbronchial and Transesophageal Fine-Needle Aspiration Using an Ultrasound Bronchoscope in Mediastinal Staging of Potentially Operable Lung Cancer. Chest 
2010;138:795-802.

27. Liberman M, Sampalis J, Duranceau A, et al. Endosonographic Mediastinal Lymph Node Staging of Lung Cancer. Chest 2014;146:389-97.

28. Herth FJF, Krasnik M, Kahn N, et al. Combined Endoscopic-Endobronchial Ultrasound-Guided FineNeedle Aspiration of Mediastinal Lymph Nodes Through a Single Bronchoscope in 150 Patients With Suspected Lung Cancer. Chest 2010;138:790-4.

29. Annema JT, Versteegh MI, Veseliç M, et al. Endoscopic Ultrasound-Guided Fine-Needle Aspiration in the Diagnosis and Staging of Lung Cancer and Its Impact on Surgical Staging. J Clin Oncol 2005;23:8357-61.

30. Annema JT, van Meerbeeck JP, Rintoul RC, et al. Mediastinoscopy vs Endosonography for Mediastinal Nodal Staging of Lung Cancer: A Randomized Trial. JAMA 2010;304:2245-52.

31. Herth FJF, Eberhardt R, Krasnik M, et al. Endobronchial Ultrasound-Guided Transbronchial Needle Aspiration of Lymph Nodes in the Radiologically and Positron Emission Tomography-Normal Mediastinum in Patients With Lung Cancer. Chest 2008;133:887-91.

32. Lee KJ, Suh GY, Chung MP, et al. Combined Endobronchial and Transesophageal Approach of an Ultrasound Bronchoscope for Mediastinal Staging of Lung

Cite this article as: Chrysikos S, Gkiozos I, Dimakou K, Zervas E, Karampitsakos T, Anyfanti M, Tzouvelekis A, Samitas K, Gaga M, Koulouris N, Vasileiadis I, Syrigos K. Clinical utility of thoracic endosonography (EBUS/EUS-b) in mediastinal staging of patients with non-small cell lung cancer: comparison with integrated $\mathrm{PET} / \mathrm{CT}$ - a real-life prospective study in Greece. J Thorac Dis 2020;12(10):5657-5666. doi: $10.21037 /$ jtd-20-1735
Cancer. PLoS One 2014;9:e91893.

33. Herth FJF, Ernst A, Eberhardt R, et al. Endobronchial ultrasound-guided transbronchial needle aspiration of lymph nodes in the radiologically normal mediastinum. Eur Respir J 2006;28:910-4.

34. de Langen AJ, Raijmakers P, Riphagen I, et al. The size of mediastinal lymph nodes and its relation with metastatic involvement: a meta-analysis. Eur J Cardiothorac Surg 2006;29:26-9.

35. Vilmann P, Clementsen PF, Colella S, et al. Combined endobronchial and esophageal endosonography for the diagnosis and staging of lung cancer: European Society of Gastrointestinal Endoscopy (ESGE) Guideline, in cooperation with the European Respiratory Society (ERS) and the European Society of Thoracic Surgeons (ESTS). Endoscopy 2015;47:545-59.

36. Bryant AS, Cerfolio RJ, Klemm KM, et al. Maximum Standard Uptake Value of Mediastinal Lymph Nodes on Integrated FDG-PET-CT Predicts Pathology in Patients with Non-Small Cell Lung Cancer. Ann Thorac Surg 2006;82:417-22.

37. Hellwig D, Graeter TP, Ukena D, et al. 18F-FDG PET for Mediastinal Staging of Lung Cancer: Which SUV Threshold Makes Sense? J Nucl Med 2007;48:1761-6. 